\title{
Electro-optical modeling of bulk heterojunction solar cells
}

Thomas Kirchartz, Bart E. Pieters, Kurt Taretto, and Uwe Rau

Citation: Journal of Applied Physics 104, 094513 (2008);

View online: https://doi.org/10.1063/1.3013904

View Table of Contents: http://aip.scitation.org/toc/jap/104/9

Published by the American Institute of Physics

\section{Articles you may be interested in}

Small molecular weight organic thin-film photodetectors and solar cells

Journal of Applied Physics 93, 3693 (2003); 10.1063/1.1534621

Two-layer organic photovoltaic cell

Applied Physics Letters 48, 183 (1986); 10.1063/1.96937

Detailed Balance Limit of Efficiency of $p$-n Junction Solar Cells

Journal of Applied Physics 32, 510 (1961); 10.1063/1.1736034

Electric field assisted dissociation of charge transfer states as a mechanism of photocarrier production The Journal of Chemical Physics 80, 4157 (1984); 10.1063/1.447243

Combined optical and electrical modeling of polymer:fullerene bulk heterojunction solar cells Journal of Applied Physics 103, 084502 (2008); 10.1063/1.2905243

Ultimate efficiency of polymer/fullerene bulk heterojunction solar cells

Applied Physics Letters 88, 093511 (2006); 10.1063/1.2181635

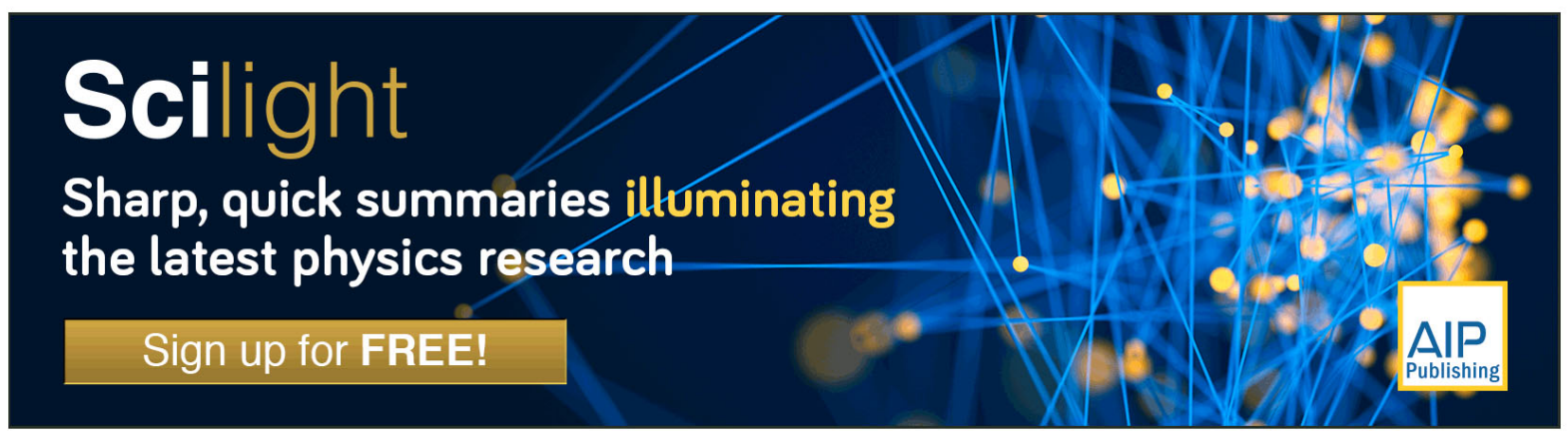




\title{
Electro-optical modeling of bulk heterojunction solar cells
}

\author{
Thomas Kirchartz, ${ }^{1, a)}$ Bart E. Pieters, ${ }^{1}$ Kurt Taretto, ${ }^{2}$ and Uwe Rau ${ }^{1}$ \\ ${ }^{1}$ IEF5-Photovoltaik, Forschungszentrum Jülich, 52425 Jülich, Germany \\ ${ }^{2}$ Departamento de Electrotecnia, Universidad Nacional del Comahue, Buenos Aires 1400, 8300 Neuquen, \\ Argentina
}

(Received 20 May 2008; accepted 20 September 2008; published online 12 November 2008)

\begin{abstract}
We introduce a model for charge separation in bulk heterojunction solar cells that combines exciton transport to the interface between donor and acceptor phases with the dissociation of the bound electron/hole pair. We implement this model into a standard semiconductor device simulator, thereby creating a convenient method to simulate the optical and electrical characteristics of a bulk heterojunction solar cell with a commercially available program. By taking into account different collection probabilities for the excitons in the polymer and the fullerene, we are able to reproduce absorptance, internal and external quantum efficiency, as well as current/voltage curves of bulk heterojunction solar cells. We further investigate the influence of mobilities of the free excitons as well as the mobilities of the free charge carriers on the performance of bulk heterojunction solar cells. We find that, in general, the highest efficiencies are achieved with the highest mobilities. However, an optimum finite mobility of free charge carriers can result from a large recombination velocity at the contacts. In contrast, Langevin-type of recombination cannot lead to finite optimum mobilities even though this mechanism has a strong dependence on the free carrier mobilities.
\end{abstract}

(C) 2008 American Institute of Physics. [DOI: 10.1063/1.3013904]

\section{INTRODUCTION}

In recent years, several new technological approaches have emerged in the field of thin film photovoltaics, some of them, like the organic bulk heterojunction (bhj) cell, ${ }^{1-4}$ using new types of device geometries. These new geometries require approaches for modeling and simulation of the devices that go beyond those used for standard inorganic device simulators. Consisting of a blend of two materials with different electron affinities, organic, inorganic, ${ }^{5}$ as well as hybrid $^{6,7}$ bhj type devices require essentially two new aspects to be added to the standard ingredients of inorganic solar cell modeling. These are the incorporations of the multistep charge separation process, starting with the creation of excitons rather than free electron/hole pairs, ${ }^{8,9}$ as well as the network of donor/acceptor interfaces needed to split the photogenerated exciton. Incorporating these effects in an electrical model leads either to complex and detailed microscopical models ${ }^{10}$ or continuum approaches ${ }^{11-13}$ that use effective parameters in order to be computationally more efficient. Some effective medium models, such as the one proposed by Koster et al. ${ }^{14}$ allow the description of generation and recombination of free charge carriers via excitons and bound electron/hole pairs as a modification of the standard generation and recombination rates, while not changing the form of the carrier continuity equations compared to the standard inorganic case.

Not only electrical but also optical modeling ${ }^{15-17}$ has recently come to the focus of research on polymer cells, for instance leading to a better interpretation of quantum efficiencies ${ }^{18,19}$ or allowing the determination of design rules for optical spacers. ${ }^{20}$ The requirements for optical simula-

${ }^{a)}$ Electronic mail: t.kirchartz@fz-juelich.de. tions differ not much between inorganic and organic thin film solar cells. The most important aspect is the ability to consider interferences in thin layers, which is usually accomplished by a matrix transfer formalism.

This article introduces an effective electrical model for bhj solar cells that is compatible with the one dimensional continuity equations for electrons and holes and with the principle of detailed balance. ${ }^{21}$ The model takes into account exciton transport, dissociation, as well as the field and temperature dependent dissociation of a bound electron/hole pair. The widely used ${ }^{22-27}$ model of Koster et al. ${ }^{14}$ taking into account only the bound pair but not the exciton diffusion, is a special case of our model. We combine our model for the generation of free carriers in bhj-solar cells with the device simulator ASA, ${ }^{28}$ which is able to calculate the generation rate of excitons with a thin film optics approach. The result is an electro-optical simulation program able to calculate, e.g., current/voltage $(J / V)$ curves, external and internal quantum efficiencies, reflectance and transmission spectra, as well as the band diagram, carrier concentrations, and electric fields in the device. As examples for the applicability of our model, we investigate the influence of mobilities of the free excitons as well as of the free charge carriers on the performance of bhj solar cells. We also show that our model reproduces experimental absorptance, quantum efficiency, and current/voltage characteristics of a polymer/fullerene device derived from literature. ${ }^{18}$

\section{ELECTRICAL MODEL}

\section{A. Charge separation scheme}

An electrical model suitable for bhj solar cells needs to consider a multistep process of current generation. Figure 1 schematically shows the path leading from an absorbed pho- 


\section{2. excitonic transport}

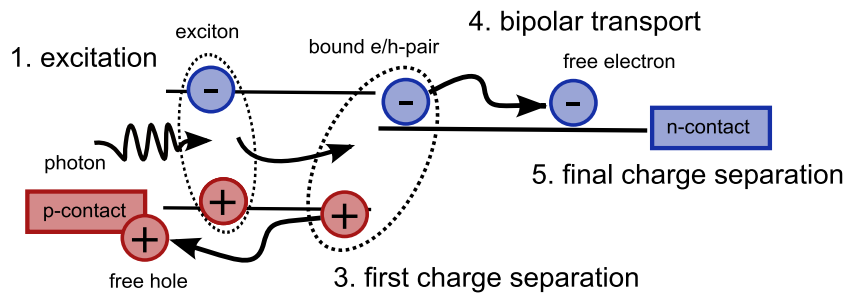

FIG. 1. (Color online) Sketch of the photoconversion process in bhj solar cells. The photon creates an exciton, which has to diffuse to the next donor/ acceptor interfaces, where it is split into a bound e/h pair. This e/h pair will then dissociate and form free charge carriers, which are transported to the contacts.

ton to the final carrier separation at the contact. In contrast to the case of inorganic solar cells, where the generation of free carriers directly follows photon absorption, in an organic solar cell an exciton is created first. Since both the exciton diffusion length and the dissociation rate of excitons in free carriers are too low for efficient charge separation in a bilayer geometry, ${ }^{29}$ the organic devices must be built up of an intimate blend of two materials. Now, the exciton has to diffuse only to the next junction between the blended materials and not directly to one of the device contacts. At the junction, the exciton is split into an electron (e) and hole (h), which are now in different phases depending on the band offsets at the junction. In the example presented in Fig. 1, the hole remains in the absorber material, which may be a polymer, while the electron is injected into the material with the higher electron affinity, which is usually formed by a $\mathrm{C}_{60}$ derivative. The electron and hole on either side of the interface are still coloumbically bound to each other. Only after this bound electron/hole $(\mathrm{e} / \mathrm{h})$ pair is split into free carriers, the bipolar drift-diffusion transport leads to the final charge separation at the contacts. Figure 2 shows how the mechanism, illustrated in Fig. 1, fits into the charge separation scheme of the complete device. At each point of the pin-type

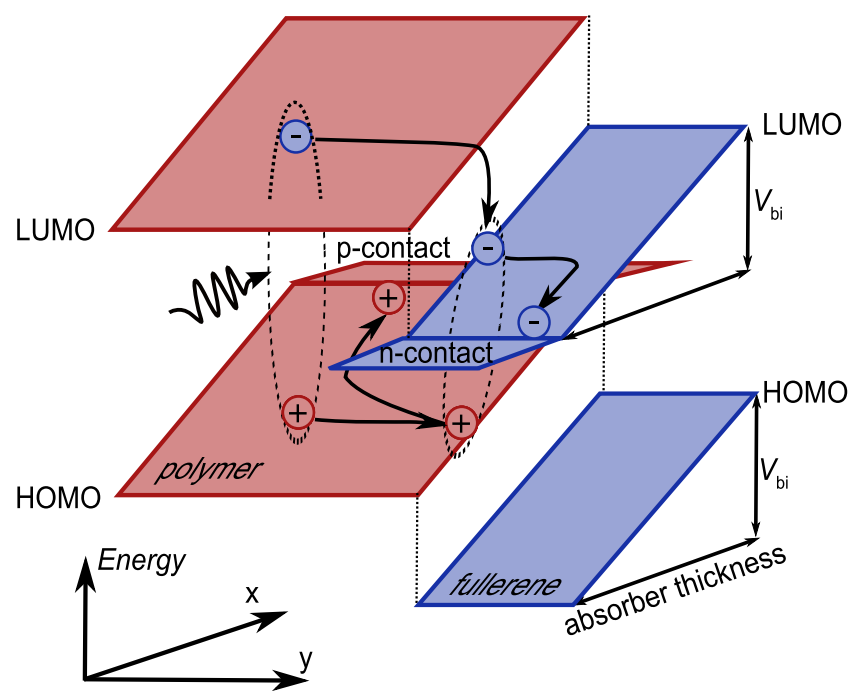

FIG. 2. (Color online) Sketch of the whole device to be simulated. At each grid point of a standard pin-junction solar cell, diffusion and dissociation of the exciton as well as dissociation of the bound e/h pair precede the creation of free e/h pairs. device, a heterojunction exists, where the processes in Fig. 1 lead to the creation of free carriers. In order to include the transport of excitons to the interface, we have to solve the continuity equation for excitons ${ }^{30-32}$ in the $y$-direction defined in Fig. 2. By solving the coupled equations for bipolar charge transport as a function of the coordinate $x$, as defined by Fig. 2, we include the transport of free carriers in the pin-type device to their respective collecting contacts.

\section{B. Differential equations for free carriers}

The scheme in Fig. 1 shows that there are two steps of charge separation and two steps of transport necessary to achieve a photocurrent. Thus, a suitable model needs to include properties of excitonic and bipolar transport, as well as properties of the splitting of the exciton and bound e/h pair and those relevant for the final charge separation. Let us start with those parts of the model we can adopt from inorganic photovoltaics.

For the simplest case of direct band to band recombination, the drift diffusion equations for the electron concentration $n$ and the hole concentration $p$ are given by

$$
\begin{aligned}
& -\frac{1}{q} \frac{d J_{n}}{d x}=-D_{n} \frac{d^{2} n}{d x^{2}}-F \mu_{n} \frac{d n}{d x}=g-k_{\mathrm{rec}} n p, \\
& \frac{1}{q} \frac{d J_{p}}{d x}=-D_{p} \frac{d^{2} p}{d x^{2}}+F \mu_{p} \frac{d p}{d x}=g-k_{\mathrm{rec}} n p,
\end{aligned}
$$

where $J_{n}$ and $J_{p}$ are the electrical electron and the hole current densities, $D_{n, p}=\mu_{n, p} k T / q$ is the diffusion constant of the electrons and holes, which depends on the electron and hole mobilities $\mu_{n}$ and $\mu_{p}$ and on the thermal voltage $k T / q$. For inorganic solar cells, the coupled solution of Eqs. (1a) and (1b) is sufficient for obtaining the carrier concentrations and subsequently the currents. In bhj solar cells, however, the properties of the generation process of free carriers at each grid point, as depicted in Fig. 2, must be included to interpret the generation rate $g$ and the recombination constant $k_{\text {rec }}$ as quantities depending on exciton diffusion and dissociation.

\section{Balance equation for bound carriers}

Figure 3 depicts the transition rates from the four possible states, photon, exciton $\chi$, bound e/h pair $\xi$, and free e/h pair. The system consists of four pairs of rates that connect the states with each other. These are (i) the generation and recombination of excitons, (ii) the dissociation of excitons forming a bound $\mathrm{e} / \mathrm{h}$ pair and the recombination of bound pairs forming an exciton, (iii) the recombination of bound pairs and the creation of bound pairs, and (iv) the dissociation of bound pairs forming free carriers and the recombination of free carriers forming bound e/h pairs. Due to the principle of detailed balance each process must be in equilibrium with its inverse process if the system is in thermodynamic equilibrium. This law allows us to eliminate one of the rate constants for each pair if we introduce the equilibrium concentrations for excitons $\chi_{0}$, bound e/h pairs $\xi_{0}$, and the intrinsic carrier concentration $n_{i}$ for free e/h pairs. For the generation and recombination of the exciton, the equilibrium generation $g_{\mathrm{opt}}^{\mathrm{eq}}=\chi_{0} / \tau_{r}$, where $\tau_{r}$ is the lifetime. The coupling 


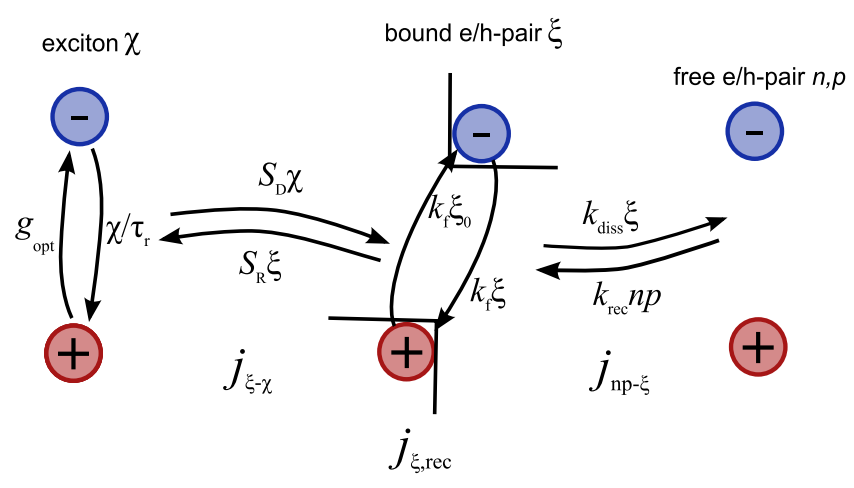

FIG. 3. (Color online) The model for modification of generation and recombination rates at each grid point in the devices includes three states of the excitation: the exciton, the bound $\mathrm{e} / \mathrm{h}$ pair and the free e/h pair. All states are connected by detailed balance rates. In addition excitons may be optically generated and excitons and bound e/h pairs may recombine.

of excitons and bound pairs leads to the relation $S_{D} \chi_{0}$ $=S_{R} \xi_{0}$, where $S_{D}$ is the dissociation and $S_{R}$ the recombination velocity. Expressing the recombination velocity in terms of the dissociation velocity allows us to write the net particle current density between excitons and bound pairs as

$$
j_{\xi \leftrightarrow \chi}=S_{D}\left(\frac{\xi}{\xi_{0}} \chi_{0}-\chi_{h i}\right),
$$

where $\chi_{h i}$ is the concentration of excitons at the heterointerface between the donor and acceptor phases. The recombination of bound pairs must again lead to a zero net generation rate of bound pairs in thermodynamic equilibrium. Thus, the net recombination current density is

$$
j_{\xi, \mathrm{rec}}=k_{f} \xi_{0}\left(\frac{\xi}{\xi_{0}}-1\right),
$$

where $k_{f}$ is the rate constant for the decay. Equation (3) means that in thermodynamic equilibrium, the generation and recombination rates of bound pairs are equal according to the principle of detailed balance and are given by $k_{f} \xi_{0}$. In nonequilibrium, the recombination rate $k_{f} \xi$ strongly increases with the increase in bound pair concentration $\xi$ while the generation rate stays the same $\left(k_{f} \xi_{0}\right)$ and becomes negligible already for small deviations from equilibrium.

The exchange between bound and free electron hole pairs leads to the balance between dissociation and recombination. We express the dissociation constant $k_{\mathrm{diss}}=k_{\mathrm{rec}} n_{i}^{2} / \xi_{0}$ as a function of the recombination constant $k_{\text {rec }}$ and receive for the net current density

$$
j_{n p \leftrightarrow \xi}=k_{\text {rec }}\left(n p-\frac{\xi}{\xi_{0}} n_{i}^{2}\right) .
$$

What we finally need, in order to compute the effective generation and recombination rates of the coupled processes shown in Fig. 3, is the result of Eq. (4), however independent of the concentration of excitons $\chi$ and bound pairs $\xi$. In order to eliminate $\xi$ in Eq. (4), we enforce current continuity between the current densities defined in Eqs. (2)-(4), leading to a balance equation

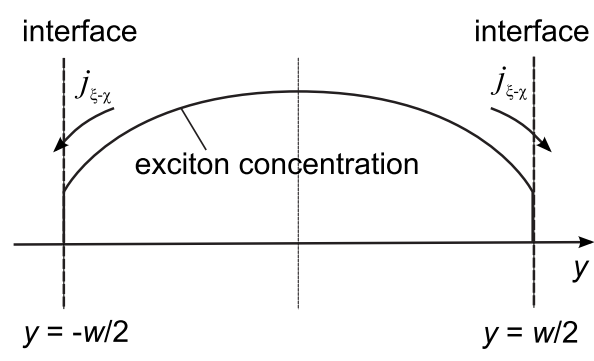

FIG. 4. Scheme of the coordinate system used for the analytical solution of the exciton diffusion equation. The generation rate is assumed to be constant over the width $w$ between two donor/acceptor interfaces.

$$
\begin{aligned}
j_{n p \leftrightarrow \xi}-j_{\xi \leftrightarrow \chi}-j_{\xi, \mathrm{rec}}= & k_{\mathrm{rec}}\left(n p-\frac{\xi}{\xi_{0}} n_{i}^{2}\right)-S_{D}\left(\frac{\xi}{\xi_{0}} \chi_{0}-\chi_{\mathrm{hi}}\right) \\
& -k_{f} \xi_{0}\left(\frac{\xi}{\xi_{0}}-1\right) .
\end{aligned}
$$

\section{Differential equation for excitons}

To obtain the effective generation and recombination rate of free carriers, we need to calculate the solution of Eq. (4), i.e., the net current $j_{n p \leftrightarrow \xi}$, which requires the knowledge of each component of Eq. (5). Let us start with the contribution from the excitons, i.e., $S_{D} \chi_{h i}$. In order to include exciton transport and dissociation we need to add another dimension to our originally one dimensional problem. Later, we will show that analytically solving the differential equation for excitons allows us to calculate effective modifications of the one-dimensional differential equations for free carriers, thereby circumventing the need for solving the coupled differential equations in two or three dimensions.

To include exciton diffusion to the next interface, we solve the one dimensional diffusion equation for the exciton concentration $\chi$

$$
0=D_{\chi} \frac{d^{2}}{d y^{2}} \chi-\frac{\chi}{\tau_{r}}+g_{\mathrm{opt}}
$$

in a new coordinate system as depicted in Figs. 2 and 4. The generation rate $g_{\text {opt }}=g_{\text {opt }}^{\text {eq }}+g_{\text {opt }}^{\text {exc }}$ is now the actual optical generation rate as calculated by a transfer matrix approach and the sum of the generation rate $g_{\text {opt }}^{\text {eq }}$ due to black body radiation of the environment and the generation rate $g_{\text {opt }}^{\text {exc }}$ due to excess illumination. In addition, $\tau_{r}$ is the lifetime of excitons and $D_{\chi}$ is the exciton diffusion constant. We assume that the generation rate is independent of the coordinate $y$, while interferences and damping are taken into account for the coordinate $x$. The boundary conditions for the particle currents at the interfaces are assumed to be symmetric

$$
j_{\xi \leftrightarrow \chi}= \pm D_{\chi} \frac{d}{d y} \chi_{h i}=S_{D} \chi_{0} \frac{\xi}{\xi_{0}}-S_{D} \chi_{h i}
$$

for $y= \pm w / 2$, where $w$ is the distance between two interfaces. The solution for the exciton concentration is

$$
\chi(y)=\left(A_{r}+A_{g}\right)\left\lfloor\cosh \left(y / L_{\chi}\right)\right\rfloor+g_{\text {opt }} \tau_{r},
$$

where $L_{\chi}=\sqrt{\mu_{\chi} \tau_{r} k T / q}$. Equation (8) superimposes two contributions to the exciton population, namely, the optical gen- 
eration proportional to the exciton generation rate $g_{\text {opt }}$

$$
A_{g}=\frac{-S_{D} g_{\mathrm{opt}} \tau_{r}}{S_{D} \cosh \left(w / 2 L_{\chi}\right)+D_{\chi} / L_{\chi} \sinh \left(w / 2 L_{\chi}\right)}
$$

and the injection and extraction of excitons via the coupling to the bound $\mathrm{e} / \mathrm{h}$ pairs $\xi$

$$
A_{r}=\frac{S_{D} \chi_{0} \xi / \xi_{0}}{S_{D} \cosh \left(w / 2 L_{\chi}\right)+D_{\chi} / L_{\chi} \sinh \left(w / 2 L_{\chi}\right)},
$$

which is thus proportional to $\xi / \xi_{0}$. Note here that due to the earlier derived relation $g_{\mathrm{opt}}^{\mathrm{eq}}=\chi_{0} / \tau_{r}, A_{r}+A_{g}=0$ holds in thermodynamic equilibrium, i.e., when $g_{\text {opt }}=g_{\text {opt }}^{\text {eq }}$.

Now, the solution for the exciton concentration as a function of $y$ enables us to calculate the current, describing the exchange between bound pairs and excitons. For each domain with length $w / 2$, the current $j_{\xi \leftrightarrow \chi}$ is defined by Eq. (7). The current $j_{\xi \leftrightarrow \chi}=j_{g}+j_{r}$ consists of two contributions, which depend on the concentration $\chi_{h i}=\chi(y= \pm w / 2)$ of excitons at the heterointerface. Again we split the concentration $\chi_{h i}=\chi_{g}+\chi_{r}$ in two parts corresponding to the concentration $\chi_{g}$ of excitons at the interface due to photogeneration

$$
\chi_{g}=A_{g} \cosh \left(\frac{w}{2 L_{\chi}}\right)+g_{\text {opt }} \tau_{r}=g_{\text {opt }} \tau_{r} \beta
$$

and to the concentration $\chi_{r}$ due to injection of bound pairs

$$
\chi_{r}=A_{r} \cosh \left(\frac{w}{2 L_{\chi}}\right)=\chi_{0} \frac{\xi}{\xi_{0}}(1-\beta) .
$$

The current due to exciton generation is then

$$
j_{g}=-S_{D} \chi_{g}=-S_{D} g_{\text {opt }} \tau_{r} \beta
$$

and the current due to the injection of bound $\mathrm{e} / \mathrm{h}$ pairs is

$$
j_{r}=S_{D}\left(\frac{\xi}{\xi_{0}} \chi_{0}-\chi_{r}\right)=S_{D} \chi_{0} \frac{\xi}{\xi_{0}} \beta
$$

where we used the abbreviation

$$
\beta=\frac{D_{\chi}}{L_{\chi}} \frac{\sinh \left(w / 2 L_{\chi}\right)}{S_{D} \cosh \left(w / 2 L_{\chi}\right)+D_{\chi}\left(L_{\chi}\right) \sinh \left(w / 2 L_{\chi}\right)} .
$$

Finally, the result for the current defined in Eq. (2) is

$$
\begin{aligned}
j_{\xi \leftrightarrow \chi}= & S_{D}\left(\frac{\xi}{\xi_{0}} \chi_{0}-\chi_{h i}\right)=S_{D}\left(\frac{\xi}{\xi_{0}} \chi_{0}-g_{\text {opt }} \tau_{r} \beta-\frac{\xi}{\xi_{0}} \chi_{0}(1\right. \\
& -\beta))=S_{D} \beta\left(\frac{\xi}{\xi_{0}} \chi_{0}-g_{\text {opt }} \tau_{r}\right) .
\end{aligned}
$$

\section{E. Effective generation and recombination rates}

In order to determine the current density $j_{n p \leftrightarrow \xi}$ and therewith the effective generation and recombination rates, we need to calculate the concentration of bound pairs. The balance equation for bound pairs, Eq. (5), leads to

$$
\frac{\xi}{\xi_{0}}=\frac{S_{D} \beta g_{\mathrm{opt}} \tau_{r}+k_{\mathrm{rec}} n p+k_{f} \xi_{0}}{S_{D} \beta \chi_{0}+k_{\mathrm{rec}} n_{i}^{2}+k_{f} \xi_{0}}
$$

in steady state. Let us briefly discuss the meaning of Eq. (17) by considering the extreme cases. If either the coupling to the free e/h pairs or the coupling to the excitons is dominant over the other and over the nonradiative decay, the Fermi level-representing the ratio of concentration to equilibrium concentration-will be equal for the two strongly coupled states. Strong coupling means a sufficiently high rate or short lifetime that the other components of the two sums in numerator and denominator are negligible. That means if (i) the recombination constant $k_{\text {rec }}$ is very high compared to the other rates, Eq. (17) will simplify to $\xi / \xi_{0}=n p / n_{i}^{2}$, while (ii) a high dissociation velocity $S_{D}$ leads to $\xi / \xi_{0}=\chi_{h i} / \chi_{0}$ $=g_{\text {opt }} \tau_{r} / \chi_{0}$. If (iii) the nonradiative decay channel is dominant, $\xi$ will approach its equilibrium value $\left(\xi / \xi_{0}=1\right)$.

Now, we insert Eq. (17) into Eq. (4) and obtain

$$
\begin{aligned}
j_{n p \leftrightarrow \xi} & =k_{\mathrm{rec}}\left(n p-\frac{\xi}{\xi_{0}} n_{i}^{2}\right) \\
& =k_{\mathrm{rec}}\left(n p-n_{i}^{2} \frac{S_{D} \beta g_{\mathrm{opt}} \tau_{r}+k_{\mathrm{rec}} n p+k_{f} \xi_{0}}{S_{D} \beta \chi_{0}+k_{\mathrm{rec}} n_{i}^{2}+k_{f} \xi_{0}}\right) \\
& =k_{\mathrm{rec}}\left(\frac{k_{f} \xi_{0}\left(n p-n_{i}^{2}\right)+S_{D} \beta\left(\left[n p-n_{i}^{2}\right] \chi_{0}-n_{i}^{2} g_{\mathrm{opt}}^{\mathrm{exc}} \tau_{r}\right)}{S_{D} \beta \chi_{0}+k_{\mathrm{rec}} n_{i}^{2}+k_{f} \xi_{0}}\right) .
\end{aligned}
$$

Note that we used the earlier derived relation $g_{\text {opt }}^{\text {eq }}=\chi_{0} / \tau_{r}$ in order to obtain Eq. (18). In general, the derivation of the particle currents $j_{x}$ in $x$-direction, i.e., the effective generation and recombination rates depend on the particle current in $y$-direction via

$$
\frac{d j_{x}}{d x}:=\tilde{g}-\tilde{R}=\frac{j_{n p \leftrightarrow \xi}}{w / 2},
$$

since the particle current was defined as the current coming from one domain with width $w / 2$. Thus, splitting the result of Eq. (19) in terms $\sim g_{\text {opt }}^{\text {exc }}$ yields the new effective generation rate to be inserted in Eqs. (1a) and (1b) and those $\sim k_{\text {rec }} n p$ yields the new effective recombination rate to be inserted in Eqs. (1a) and (1b). The new effective generation rate is then

$$
\tilde{g}=g_{\mathrm{opt}}^{\operatorname{exc}} \frac{S_{D} \beta \tau_{r}}{w / 2} \frac{k_{\mathrm{rec}} n_{i}^{2}}{S_{D} \beta \chi_{0}+k_{\mathrm{rec}} n_{i}^{2}+k_{f} \xi_{0}}
$$

and the new effective recombination rate is

$$
\tilde{R}=\frac{k_{\mathrm{rec}}}{w / 2}\left(1-\frac{k_{\mathrm{rec}} n_{i}^{2}}{S_{D} \beta \chi_{0}+k_{\mathrm{rec}} n_{i}^{2}+k_{f} \xi_{0}}\right)\left(n p-n_{i}^{2}\right) .
$$

\section{F. Equilibrium concentration of excitons}

There still remain some open questions, especially how to choose the value of $k_{\text {rec }}$ and how to determine the equilibrium concentrations $\chi_{0}, \xi_{0}$, and $n_{i}$ of the different particles. For efficient charge separation at the interface between donor and acceptor phases, band offsets are necessary. In our model, these band offsets are implicitly defined through the 
value of the equilibrium concentration of excitons $\chi_{0}$. For a given density of states, the energy of the thermalized exciton is the only relevant parameter affecting the equilibrium concentration. Thus, a high energy difference $\Delta E$ between the lowest unoccupied molecular orbital (LUMO) of the donor and the LUMO of the acceptor leads to efficient charge separation, which is reflected in our model by a low $\chi_{0}$ and subsequently to less back transfer of bound e/h pairs in excitons. Since the exciton density of states is an unknown parameter, we cannot give quantitative values for $\chi_{0}$ e.g., as a function of exciton energy in the polymer. Instead, for our simulations, we chose the value of $\chi_{0}$ to be sufficiently low that the back transfer of bound e/h pairs in excitons does not lead to an efficiency decrease. This is the case, when $S_{D} \beta \chi_{0}$ $\ll k_{\text {rec }} n_{i}^{2}+k_{f} \xi_{0}$ is satisfied, since for this condition, the mathematical expression for recombination rate [Eq. (21)] is independent of $S_{D} \beta \chi_{0}$, meaning that the recombination of free carriers does not depend on injection and recombination of excitons.

\section{G. Comparison with the model of Koster et al. (Ref. 14)}

Our model describes both, finite collection of excitons as well as the dissociation of the bound e/h-pair. If we only consider the latter part, the influence of the bound pair on the generation and recombination, we describe the same physical mechanisms as the model of Koster et al., ${ }^{14}$ which we will refer to as the KSMB model (see Ref. 14). To make our model equivalent to the KSMB model for the case of efficient exciton collection, we need to choose the values of $k_{\text {rec }}$ and $\xi_{0}$ appropriately.

We define the recombination constant ${ }^{33}$

$$
k_{\mathrm{rec}}=\frac{q \min \left(\mu_{n}, \mu_{p}\right)}{\varepsilon_{0} \varepsilon_{r}}(w / 2)
$$

according to a slightly modified Langevin recombination rate. We have to include the domain width $w / 2$ to ensure that $\left(k_{\mathrm{rec}} n_{i}^{2}\right)=\mathrm{cm}^{-2} \mathrm{~s}^{-1}$. Note here that also the decay rate constant $k_{f}$ has a modified unit, namely, $\left(k_{f}\right)=\mathrm{cm}^{-1} \mathrm{~s}^{-1}$ again to ensure that $\left(k_{f} \chi_{0}\right)=\mathrm{cm}^{-2} \mathrm{~s}^{-1}$. Like the recombination rate constant also the decay rate constant relates to the rate constant $k_{f, \mathrm{KSMB}}$ in the KSMB model via $k_{f}=k_{f, \mathrm{KSMB}} w / 2$.

The detailed balance requires that in thermodynamic equilibrium, the net current $j_{n p \leftrightarrow \xi}$ vanishes, leading to the requirement $k_{\mathrm{diss}}=k_{\mathrm{rec}} n_{i}^{2} / \xi_{0}$ for the dissociation rate of bound pairs. We already used this equality for the derivation of the effective generation and recombination rates, allowing us to express these rates independently of the value of $k_{\mathrm{diss}}$. If we use the expression for $k_{\text {diss }}$ that was suggested by the KSMB model, ${ }^{14}$ the relation $k_{\text {diss }}=k_{\text {rec }} n_{i}^{2} / \xi_{0}$ allows us to fix the equilibrium concentration for bound $\mathrm{e} / \mathrm{h}$ pairs as the only left unknown. This operation leads to

$$
\xi_{0}=\frac{4 \pi z^{3} \exp \left(E_{B}(a) / k T\right) n_{i}^{2}}{3 J_{1}(2 \sqrt{-2 b}) / \sqrt{-2 b}},
$$

where $J_{1}(2 \sqrt{-2 b}) / \sqrt{-2 b}=1+b+b^{2} / 3+b^{3} / 18+\cdots$ is the first order Bessel function,

$$
b=\frac{q^{3} F}{8 \pi \varepsilon_{0} \varepsilon_{r}(k T)^{2}},
$$

where $F$ is the electric field, $\varepsilon=\varepsilon_{0} \varepsilon_{r}$ is the dielectric constant, $a$ is the separation distance between the bound electron and hole, and $E_{B}(a)=q^{2} / 4 \pi \varepsilon a$ is the binding energy of the bound e/h pair. As shown in Refs. 14 and 22, the definitions in Eqs. (23) and (24) imply a strong field and therefore bias dependence of bound e/h-pair dissociation, which has a considerable influence on the fill factor of the device. Using the definitions in Eqs. (22)-(24) and assuming $S_{D} \beta \chi_{0} \ll k_{\text {rec }} n_{i}^{2}+k_{f} \xi_{0}$, the dissociation probability

$$
p_{\mathrm{diss}}=\frac{k_{\mathrm{rec}} n_{i}^{2}}{S_{D} \beta \chi_{0}+k_{\mathrm{rec}} n_{i}^{2}+k_{f} \xi_{0}}
$$

appearing in Eqs. (20) and (21) is the same as in the KSMB model. The generation rate from Eq. (20) is now

$$
\tilde{g}=g_{\text {opt }} p_{\text {diss }} 2 \tau_{r} \beta S_{D} / w=\tilde{g}_{\mathrm{KSMB}} 2 \tau_{r} \beta S_{D} / w=: \widetilde{g}_{\mathrm{KSMB}} f_{c},
$$

and thus being a series connection of the generation rate $\tilde{g}_{\text {KSMB }}$ as defined by Ref. 14 and of a collection efficiency $f_{c}$ defined as the probability that a photogenerated exciton contributes to the current. Note that we omitted the integration over a distribution of separation distances, as carried out in Ref. 14, since it is incompatible with the principle of detailed balance. Thus, the generation rate $\widetilde{g}_{\text {KSMB }}$ represents the generation rate from Ref. 14 without this integration.

Especially for the simulation of $J / V$ curves, the recombination rate is of high importance. For all following simulations, we modify the recombination rate as defined in Eq. (21) in the same way as the generation rate. Using the dissociation probability for bound $\mathrm{e} / \mathrm{h}$ pairs, the recombination rate becomes

$$
\widetilde{R}=k_{\text {rec }} n p\left(1-p_{\text {diss }}\right)
$$

with $p_{\text {diss }}$ defined by Eq. (25). Since we assume the back reaction of bound $\mathrm{e} / \mathrm{h}$ pairs into excitons to be weak, the recombination constant is identical to the one derived by Ref. 14.

\section{SIMULATION OF MOBILITY EFFECTS}

\section{A. Influence of the carrier mobilities and the surface recombination velocity}

The KSMB model has several implications for the $J / V$ curve and performance of the device. Among the most prominent are the temperature and field dependence of the photocurrent and the dependence of generation and recombination rate on the mobility of the free charge carriers. While the field and temperature dependence of the photocurrent have already been extensively discussed, ${ }^{22,24}$ the influence of the mobility on the solar cell efficiency is still under debate. Marsh et al., ${ }^{10}$ for instance, point out that high mobilities are of particular importance for organic solar cells, while Mandoc et $a l^{34}$ use the KSMB model to show that solar cell efficiency has a maximum for finite mobilities and decreases for higher mobilities. These findings lead to the question if 
and under which circumstances a high mobility deteriorates device performance - in our model and in general.

For this discussion on the charge carrier mobility, let us simplify our model in a way that the dependencies of generation and recombination rate on mobility become apparent. Let us therefore set the exciton collection efficiency $f_{c}=1$ and let us neglect all field dependencies. Then the generation rate is

$$
\widetilde{g}=\frac{g_{\mathrm{opt}}^{\mathrm{exc}}}{w / 2}\left(\frac{k_{\mathrm{rec}} n_{i}^{2}}{k_{\mathrm{rec}} n_{i}^{2}+k_{f} \xi_{0}}\right)
$$

and the recombination rate is

$$
\widetilde{R}=\frac{\left(n p-n_{i}^{2}\right)}{w / 2}\left(\frac{k_{\mathrm{rec}} k_{f} \xi_{0}}{k_{\mathrm{rec}} n_{i}^{2}+k_{f} \xi_{0}}\right)
$$

It can be seen that both increase monotonically with $k_{\text {rec }}$ and, thus, due to the Langevin equation [see Eq. (23)] with the mobility of the slowest carrier. Although the recombination rate increases with mobility, the ratio

$$
\frac{\widetilde{g}}{\widetilde{R}}=\frac{g_{\mathrm{opt}}^{\mathrm{exc}}}{\left(n p-n_{i}^{2}\right)} \frac{n_{i}^{2}}{k_{f} \xi_{0}}
$$

is independent of $k_{\text {rec }}$ and of carrier mobility and thus, one would not expect a decrease in open circuit voltage with increasing carrier mobility as reported in Ref. 34.

At this point it becomes important to consider the boundary conditions between absorber and contacts. An infinite surface recombination velocity as assumed in Ref. 34 for the respective minorities will lead to strongly increased recombination at the surfaces if the mobilities are high. Thus, low mobilities act like a passivation for infinitely defective surfaces. However, infinite surface recombination is not a fundamental aspect of organic solar cells that is necessary for device functionality but instead an additional sink for minority carriers, which may become important for future device generations.

Figure 5(a) shows the short circuit current density $J_{s c}$ as a function of mobility for different surface recombination velocities $S_{C}=0,10^{2}, 10^{4}, 10^{6} \mathrm{~cm} / \mathrm{s}$, and $S_{C}=\infty$, which corresponds to the case discussed in Ref. 34. The short circuit current increases monotonically with mobility. The saturation level depends on surface recombination velocity since it affects the amount of recombination at short circuit.

Figure 5(b) shows the open circuit voltage, which decreases monotonically with mobility if the surface is not perfectly passivated $\left(S_{C}=0\right)$. Figure 5 (c) shows the efficiency as a function of mobility for different surface recombination velocities. The simulations with $S_{C}=0$ and $10^{2} \mathrm{~cm} / \mathrm{s}$ show a monotonous increase in efficiency with mobility. Very high surface recombination velocities of $S_{C}=10^{6} \mathrm{~cm} / \mathrm{s}$ lead to a dip in efficiency by around $1 \%$ absolute for very high mobilities. The parameters used for the simulation in Fig. 5 are a constant generation rate $g_{\mathrm{opt}}^{\mathrm{exc}}=6 \times 10^{21} \mathrm{~cm}^{-3}$, an intrinsic carrier concentration $n_{i}=3.5 \times 10^{8} \mathrm{~cm}^{-3}$, the equilibrium decay rate $k_{f} \xi_{0} /(w / 2)=6 \times 10^{6} \mathrm{~cm}^{-3} \mathrm{~s}^{-1}$ for the bound pairs, a built in voltage $V_{b i, 0}=1.1 \mathrm{~V}$, a ratio of electron and hole mobility $\mu_{n} / \mu_{p}=10$, and an absorber thickness $w=100 \mathrm{~nm}$.

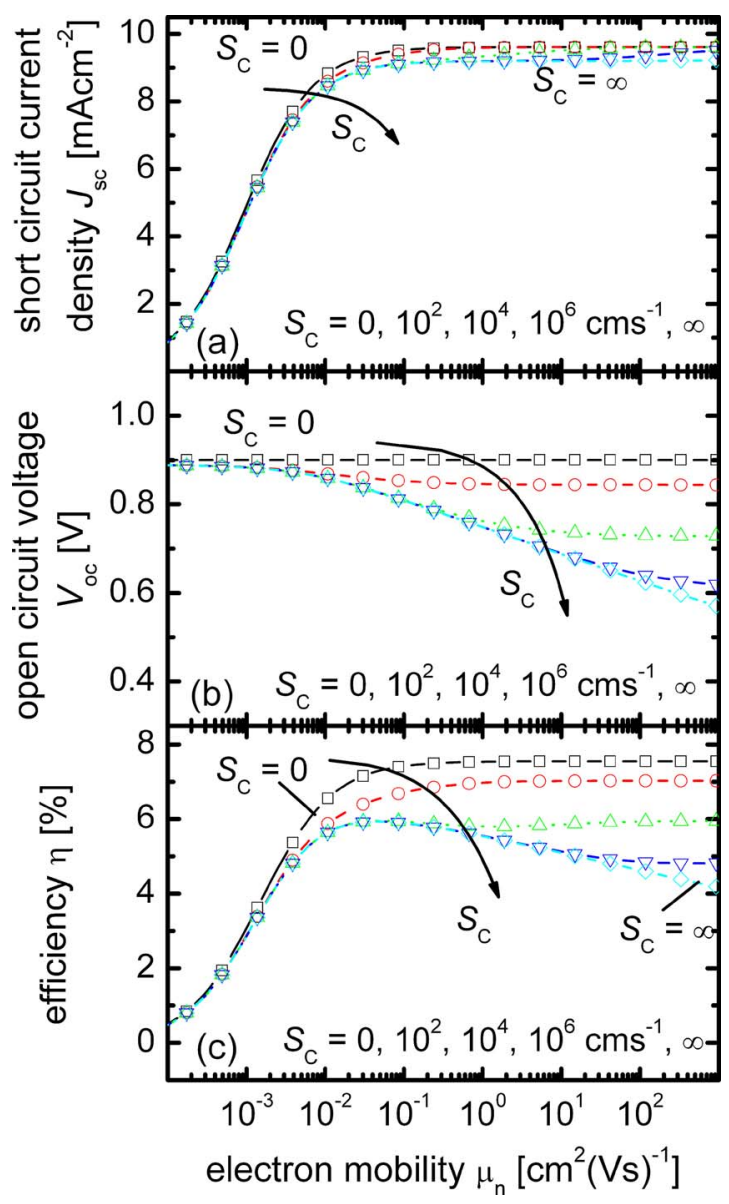

FIG. 5. (Color online) (a) Short circuit current, (b) open circuit voltage, and (c) solar cell efficiency as a function of electron mobility $\mu_{n}$ for different values of the recombination velocity $S_{C}$ at the absorber/contact interface. For well passivated surfaces, the efficiency increases monotonically with mobility. Only for high recombination at the interface, the efficiency benefits from low mobilities, since they effectively passivate the defective surface regions. The parameters used are a constant generation rate $g_{\mathrm{opt}}^{\mathrm{exc}}=6$ $\times 10^{21} \mathrm{~cm}^{-3}$, an intrinsic carrier concentration $n_{i}=3.5 \times 10^{8} \mathrm{~cm}^{-3}$, the equilibrium decay rate $k_{f} \xi_{0} /(w / 2)=6 \times 10^{6} \mathrm{~cm}^{-3} \mathrm{~s}^{-1}$ for the bound pairs, a built in voltage $V_{b i, 0}=1.1 \mathrm{~V}$, a ratio of electron and hole mobility $\mu_{n} / \mu_{p}=10$, and an absorber thickness $w=100 \mathrm{~nm}$.

The thickness of the device has an important influence on the outcome of the simulation presented in Fig. 5. A thicker device will impose stricter requirements for the mobility that has to be reached to ensure perfect collection of carriers. Thus, the mobility where the short circuit current starts to drop below its maximum will increase with increasing thickness. In addition, the relative influence of bulk to surface recombination changes with thickness. For thicker devices, the volume recombination contributes a larger share to the total recombination current as for thinner devices.

In general, we conclude that a high mobility cannot be detrimental for the device performance as long as it only influences the charge separation path crucial for the photovoltaic effect. ${ }^{35}$ Due to the principle of detailed balance, enhanced recombination of free carriers to bound carriers will always imply more efficient dissociation, i.e., a better coupling between the free and the bound pairs. The higher the coupling, the smaller the losses during charge separation will be, while the fact that this coupling also increases recombination can never lead to a performance loss. 


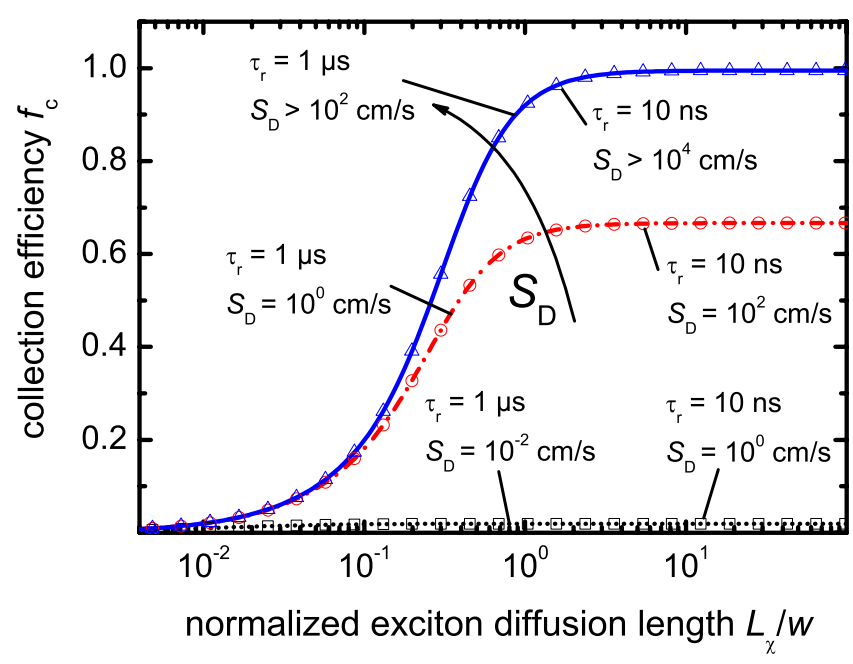

FIG. 6. (Color online) Collection efficiency $f_{c}$ of excitons displayed as a function of the exciton diffusion length $L_{\chi}$ normalized to the distance $w$ between two donor/acceptor interfaces using the exciton dissociation velocity $S_{D}$ and the exciton lifetime $\tau_{r}$ as parameters. For efficient collection two requirements must be fulfilled. The transport to the interface must be efficient $\left(L_{\chi} / w>1\right)$ and the interface dissociation must be faster than the recombination of the excitons $\left(\tau_{r} S_{D} \gg w\right)$. The average distance to the next interface is chosen to be $w=10 \mathrm{~nm}$.

However, increased mobilities can enhance recombination via additional (parasitic) pathways like high recombination at the absorber/contact interface. Thus, passivation of such defective regions is in theory also possible by making the transport toward these defective regions less likely, i.e., by decreasing the mobility. However, such an effect is neither caused by the Langevin type recombination rate nor is it a specific feature of organic and/or bhj solar cells.

\section{B. Influence of exciton diffusion on the photocurrent}

Figure 6 shows the result of calculating $f_{c}=2 \tau_{r} S_{D} \beta / w$ as a function of exciton diffusion length $L_{\chi}$ normalized to the average distance $w$ between two interfaces. Apart from the exciton diffusion length and thus the exciton mobility, the collection efficiency also depends on the exciton lifetime $\tau_{r}$ and the exciton dissociation velocity $S_{D}$. Therefore, we use both quantities as parameters and show the results for $\tau_{r}$ $=1 \mu \mathrm{s}$ (lines) and for $\tau_{r}=10 \mathrm{~ns}$ (symbols). In order to have an efficient collection of excitons, i.e., $f_{c} \approx 1$, the transport of excitons to the next heterointerface between donor and acceptor phases as well as the dissociation velocity at this interface must be high. Thus, for sufficiently high $S_{D}$ and ratios $L_{\chi} / w \gg 1$, the collection efficiency $f_{c}$ in Fig. 6 approaches unity, which corresponds to the physical case, where each photogenerated exciton creates one bound e/h pair. For low values of the dissociation velocity $S_{D}$ the collection efficiency $f_{c}$ saturates at a lower level for a high ratio $L_{\chi} / w$ $\gg 1$. For lower ratios $L_{\chi} / w \ll 1$, the excitons do not reach the next interface but recombine instead. The collection efficiency is then reduced below one even for high dissociation velocities.

From the two data sets for $\tau_{r}=1 \mu$ s (lines) and for $\tau_{r}$ $=10 \mathrm{~ns}$ (symbols), we learn that the critical ratios $L_{\chi} / w$, where the short circuit current starts to drop below its maximum, are independent of the choice of $\tau_{r}$ or $S_{D}$. However, the surface recombination velocity required for a certain short circuit current at high $L_{\chi} / w$ depends critically on the assumed lifetime $\tau_{r}$. We chose the surface recombination velocities in a way that we have three pairs of constant $\tau_{r} S_{D}$. The good agreement of the pairs indicates that the collection efficiency depends mostly on this product as long as $L_{\chi} / w$ is kept the same. Analytically, this becomes obvious, when calculating the collection efficiency for $L_{\chi} / w \rightarrow \infty$, leading to $f_{c}=\left(1+w / 2 S_{D} \tau_{r}\right)^{-1}$.

\section{APPLICATION TO EXPERIMENTAL DATA}

In order to compare the model to experimental results from literature, we implemented it in a commercial device simulator. The device simulator chosen here is called ASA (Ref. 28) and is mostly used for thin film silicon solar cells. Due to the field dependence of the rates for the bound pairs, it is necessary to compute the optical data once and the electrical simulations several times until convergence is reached. However, ASA is flexible enough to implement such an iterative modification of input parameters without the need to change the code of ASA itself. More details about the implementation are outlined in the Appendix.

Recent investigations ${ }^{18,19}$ on the internal quantum efficiency $Q_{i}$ in bhj solar cells revealed different spectral regions with distinct differences in the internal quantum efficiency. The internal quantum efficiency has been defined by

$$
Q_{i}(E)=\frac{Q_{e}(E)}{A(E)},
$$

where $Q_{e}$ is the measured external quantum efficiency and $A(E)$ is the absorptance calculated with a matrix transfer formalism. For higher energies, the internal quantum efficiency was reported to be considerably higher than the external one. The two suggested explanations ${ }^{19}$ were that the change in $Q_{i}$ is induced by the photons being absorbed in the fullerene phase that has either (i) a smaller domain size compared to the polymer phase ${ }^{36,37}$ or (ii) a higher exciton diffusion length, which can reach values of $40 \mathrm{~nm}$ in the fullerene ${ }^{38}$ compared to $10 \mathrm{~nm}$ in the polymer.

In the framework of our model, both explanations have nearly the same effect. Under the assumption that the transfer of excitons at the interface into bound e/h pairs at the interface is efficient $\left(S_{D}>10^{2} \mathrm{~cm} / \mathrm{s}\right)$, smaller domain sizes and higher exciton diffusion lengths both increase the ratio $L_{\chi} / w$ and subsequently the collection efficiency $f_{c}$. For exciton diffusion lengths as high as $40 \mathrm{~nm}$, it is reasonable to assume that $L_{\chi} / w \gg 1$ holds in the fullerene and, thus, that the collection efficiency in the fullerene $f_{c, F} \approx 1$. This finding helps to adjust the value of the generation rate $\widetilde{g}_{\text {KSMB }}$ accounting for bound e/h-pair dissociation, which is the same for excitons created in the fullerene and in the polymer. The internal quantum efficiency for lower energies, corresponding to absorption in the polymer, then allows us to fix the collection efficiency in the polymer $f_{c, P}<1$. The other parameters of the model, especially those entering in the dissociation probability $P_{\text {diss }}$ for bound e/h pairs have to be fixed by comparison between simulated and experimental current/voltage $(J / V)$ curve. 


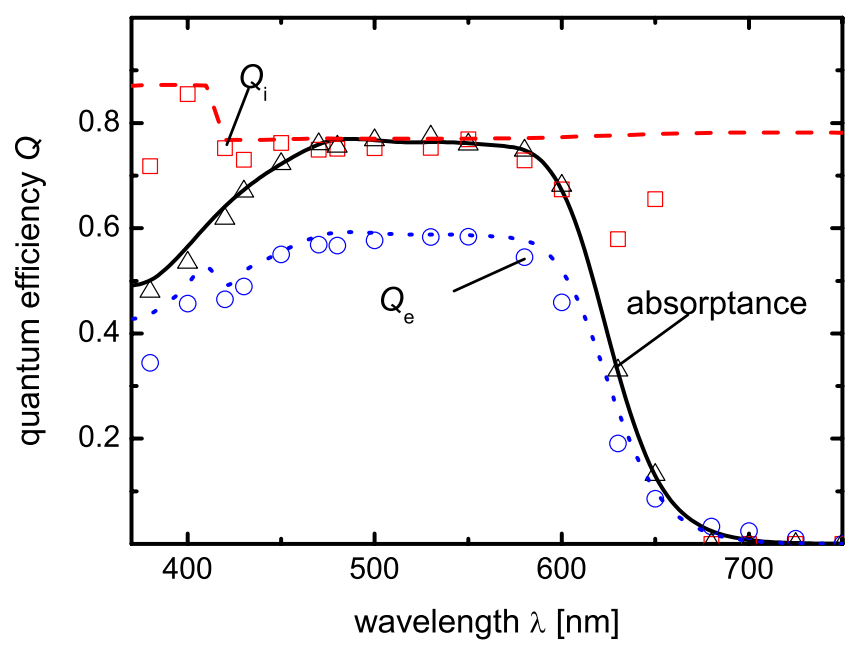

FIG. 7. (Color online) Internal and external quantum efficiencies as well as absorptance of the cell presented in Ref. 18 (open symbols) compared to our simulations (lines). The parameters used for the simulations were a band gap $E_{g}=1.4 \mathrm{eV}$, a built in voltage $V_{b i, 0}=E_{g} / q=1.4 \mathrm{~V}$, an effective density of states $N_{C, V}=2.5 \times 10^{19} \mathrm{~cm}^{-3}$ in valence and conduction band, a dielectric constant $\varepsilon_{r}=3.4$ of the blend, an electron mobility $\mu_{n}=1.2 \times 10^{-3} \mathrm{~cm}^{2} / \mathrm{V} \mathrm{s}$, a hole mobility $\mu_{p}=1.7 \times 10^{-4} \mathrm{~cm}^{2} / \mathrm{V} \mathrm{s}$, a decay constant for bound $\mathrm{e} / \mathrm{h}$ pairs $k_{f} /(w / 2)=2.8 \times 10^{5} \mathrm{~s}^{-1}$, and an average separation distance of bound $\mathrm{e} / \mathrm{h}$ pairs $a=1.8 \mathrm{~nm}$.

Figure 7 shows an example for the application of our model to quantum efficiency data published in Ref. 18. The open symbols represent the original data we wanted to reproduce with our simulations (lines). The layer stack needed for the optical simulations consists of $1 \mathrm{~mm}$ quartz superstrate, a total of $133 \mathrm{~nm}$ ITO, $68 \mathrm{~nm}$ of PEDOT:PSS, $186 \mathrm{~nm}$ of the absorber blend consisting of PF10TBT/PCBM (poly[9,9didecanefluorene-alt-(bis-thienylene) benzothiadiazole]/ ([6,6]-phenyl C60 butyric acid methyl ester), $1 \mathrm{~nm} \mathrm{LiF,} \mathrm{and} \mathrm{a}$ $100 \mathrm{~nm}$ thick Al back reflector. The nk-data files used in our simulations were obtained from the authors of Ref. 18 leading to the absorptance represented by the solid line in Fig. 7. First we adjusted the parameters for the exciton dissociation in the fullerene $(\lambda<420 \mathrm{~nm})$ to $L_{\chi} / w \gg 1$ and in the polymer $(\lambda>420 \mathrm{~nm})$ to $L_{\chi} / w=0.78$. All other parameters had to be adjusted by both considering the quantum efficiency as well as the whole $J / V$ curve as shown in Fig. 8 (circles are the data from Ref. 18 and the solid line represents the simulation). Among the parameters relevant for electron hole transport as well as dissociation and recombination of bound pairs, some were kept constant or within close boundaries, while others were used to obtain a good fit of the $J / V$ curve. The effective density of states $N_{C, V}=2.5 \times 10^{19} \mathrm{~cm}^{-3}$ in valence and conduction band, as well as the dielectric constant $\varepsilon_{r}=3.4$, were assumed to be the same as in Ref. 14 . The band gap $E_{g}=1.4 \mathrm{eV}$, meaning the energy difference between the LUMO in the electron acceptor (PCBM) and the highest occupied molecular orbital in the electron donor (PF10TBT) was only varied slightly around the approximate value $E_{g}$ $=1.3 \mathrm{eV}$, given by Moet et al $^{39}$ for the material system under investigation, in order to adjust the open circuit voltage precisely. For simplicity, the built in voltage was assumed to equal the band gap $V_{b i, 0}=E_{g} / q=1.4 \mathrm{~V}$. The parameters that were obtained after fitting are an electron mobility $\mu_{n}=1.2$

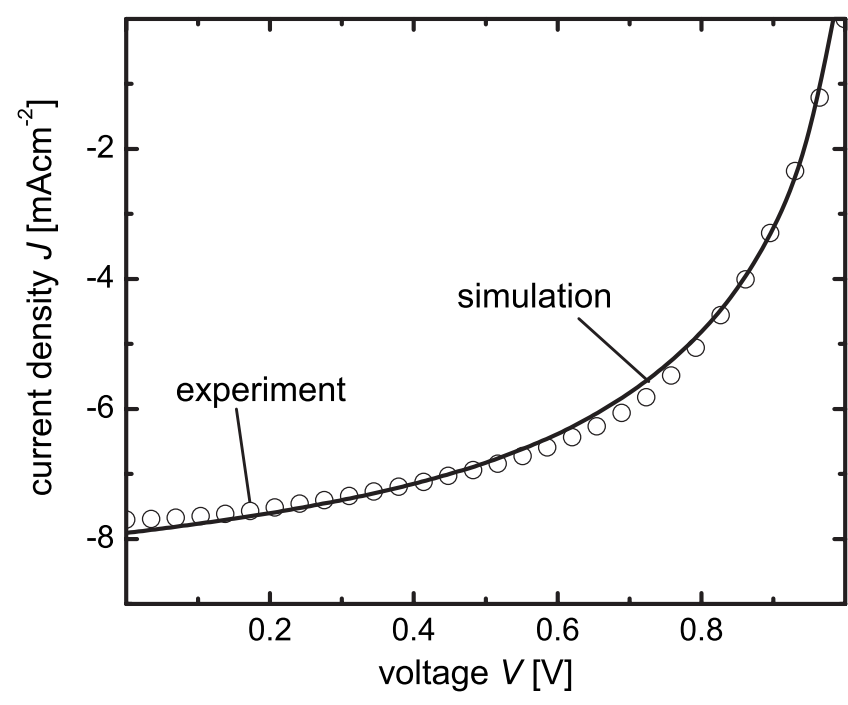

FIG. 8. Experimental current/voltage curve from Ref. 18 (open circles) compared to our simulation (solid line) using the same parameters as for the quantum efficiency in Fig. 7.

$\times 10^{-3} \mathrm{~cm}^{2} / \mathrm{V} \mathrm{s}$, a hole mobility $\mu_{p}=1.7 \times 10^{-4} \mathrm{~cm}^{2} / \mathrm{V} \mathrm{s}, \mathrm{a}$ decay constant for bound e/h pairs $k_{f} /(w / 2)=2.8 \times 10^{5} \mathrm{~s}^{-1}$, and an average separation distance of bound $\mathrm{e} / \mathrm{h}$ pairs $a$ $=1.8 \mathrm{~nm}$.

\section{CONCLUSIONS}

We have introduced a model that describes both exciton transport to the interface between donor and acceptor phases in a bhj cell as well as dissociation of the bound e/h pair at the interface. The model is applicable to standard solar cell simulators originally designed for inorganic solar cells. Herewith, we obtain a versatile method to simulate the optical and electrical characteristics of a bhj solar cell with one commercially available program that allows to iteratively modify its input file. We show how to apply our model to both quantum efficiency and current/voltage measurements. Our investigation of mobility effects of the free excitons and free charge carriers on the device performance shows that the highest solar cell efficiencies are achieved with the highest mobilities. A finite optimum mobility of free charge carriers as that observed in Ref. 34 results from surface recombination whereas Langevin type of recombination cannot lead to such an optimum below infinity.

\section{ACKNOWLEDGMENTS}

We would like to thank L. J. A. Koster, C. Deibel, and M. Kemerink for helpful discussions, L. H. Slooff for discussions and for providing the nk-data files used for Fig. 7, and A. Petersen for carefully reading the manuscript.

\section{APPENDIX: DETAILS OF THE SIMULATION PROCEDURE}

The simulation combines the software ASA, which is responsible for solving the continuity and Poisson equations and for calculating the generation rate with a matrix transfer formalism, with the software OCTAVE, ${ }^{40}$ which allows to calculate the modifications of generation and recombination 
rates as well as to make loops varying one parameter. The usual procedure for simulating quantum efficiency or $J / V$ curves starts with the definition of all parameters including thicknesses and complex refractive indices of the layer stack. ASA is then called from an OCTAVE script (code available from the authors upon request) to calculate only the optical generation $g_{\text {opt }}$ rate independent from all electrical parameters. Subsequently, OCTAVE calculates the modified generation and recombination rates assuming a constant field for the first guess. Then OCTAVE calls ASA iteratively to calculate the electric fields, every time recalculating the modifications for generation and recombination rate until convergence is reached. This has to be repeated for every voltage step.

${ }^{1}$ N. S. Sariciftci, L. Smilowitz, A. J. Heeger, and F. Wudl, Science 258 1474 (1992).

${ }^{2}$ G. Yu, J. Gao, J. C. Hummelen, F. Wudl, and A. J. Heeger, Science 270, 1789 (1995).

${ }^{3}$ H. Hoppe and N. S. Sariciftci, J. Mater. Res. 19, 1924 (2004).

${ }^{4}$ P. W. M. Blom, V. D. Mihailetchi, L. J. A. Koster, and D. E. Markov, Adv. Mater. (Weinheim, Ger.) 19, 1551 (2007).

${ }^{5}$ I. Gur, N. A. Fromer, M. L. Geier, and A. P. Alivisatos, Science 310, 462 (2005).

${ }^{6}$ W. U. Huynh, J. J. Dittmer, and A. P. Alivisatos, Science 295, 2425 (2002).

${ }^{7}$ W. J. E. Beek, M. M. Wienk, M. Kemerink, X. N. Yang, and R. A. J. Janssen, J. Phys. Chem. B 109, 9505 (2005).

${ }^{8}$ B. A. Gregg and M. C. Hanna, J. Appl. Phys. 93, 3605 (2003).

${ }^{9}$ B. A. Gregg, J. Phys. Chem. B 107, 4688 (2003).

${ }^{10}$ R. A. Marsh, C. Groves, and N. C. Greenham, J. Appl. Phys. 101, 083509 (2007).

${ }^{11}$ S. Lacic and O. Inganäs, J. Appl. Phys. 97, 124901 (2005).

${ }^{12}$ C. M. Martin, V. M. Burlakov, and H. E. Assender, Sol. Energy Mater. Sol. Cells 90, 900 (2006).

${ }^{13}$ C. M. Martin, V. M. Burlakov, H. E. Assender, and D. A. R. Barkhouse, J. Appl. Phys. 102, 104506 (2007).

${ }^{14}$ L. J. A. Koster, E. C. P. Smits, V. D. Mihailetchi, and P. W. M. Blom, Phys. Rev. B 72, 085205 (2005).

${ }^{15}$ L. A. A. Pettersson, L. S. Roman, and O. Inganäs, J. Appl. Phys. 86, 487 (1999).

${ }^{16}$ L. A. A. Pettersson, L. S. Roman, and O. Inganäs, J. Appl. Phys. 89, 5564 (2001).
${ }^{17}$ H. Hoppe, N. Arnold, N. S. Sariciftci, and D. Meissner, Sol. Energy Mater. Sol. Cells 80, 105 (2003).

${ }^{18}$ L. H. Slooff, S. C. Veenstra, J. M. Kroon, D. J. D. Moet, J. Sweelsen, and M. M. Koetse, Appl. Phys. Lett. 90, 143506 (2007).

${ }^{19}$ G. Dennler, K. Forberich, M. C. Scharber, C. J. Brabec, I. Tomis, K. Hingerl, and T. Fromherz, J. Appl. Phys. 102, 054516 (2007).

${ }^{20}$ J. Gilot, I. Barbu, M. Wienk, and R. A. J. Janssen, Appl. Phys. Lett. 91, 113520 (2007).

${ }^{21}$ P. W. Bridgman, Phys. Rev. 31, 101 (1928).

${ }^{22}$ V. D. Mihailetchi, L. J. A. Koster, J. C. Hummelen, and P. W. M. Blom, Phys. Rev. Lett. 93, 216601 (2004).

${ }^{23}$ D. W. Sievers, V. Shrotriya, and Y. Yang, J. Appl. Phys. 100, 114509 (2006).

${ }^{24}$ H. H. P. Gommans, M. Kemerink, J. M. Kramer, and R. A. J. Janssen, Appl. Phys. Lett. 87, 122104 (2005).

${ }^{25}$ L. J. A. Koster, V. D. Mihailetchi, R. Ramaker, and P. W. M. Blom, Appl. Phys. Lett. 86, 123509 (2005).

${ }^{26}$ L. J. A. Koster, V. D. Mihailetchi, and P. W. M. Blom, Appl. Phys. Lett. 88, 093511 (2006).

${ }^{27}$ J. D. Kotlarski, P. W. M. Blom, L. J. A. Koster, M. Lenes, and L. H. Slooff, J. Appl. Phys. 103, 084502 (2008)

${ }^{28}$ M. Zeman, J. van den Heuvel, B. E. Pieters, M. Kroon, and J. Willemen, Advanced Semiconductor Analysis (TU Delft, 2003).

${ }^{29}$ J. Wagner, T. Fritz, and H. Böttcher, Phys. Status Solidi A 136, 423 (1993).

${ }^{30}$ R. Corkish, D. S. P. Chan, and M. A. Green, J. Appl. Phys. 79, 195 (1996).

${ }^{31}$ M. Burgelman and B. Minnaert, Thin Solid Films 511-512, 214 (2006).

${ }^{32}$ T. Kirchartz and U. Rau, Thin Solid Films 516, 7144 (2008).

${ }^{33}$ L. J. A. Koster, V. D. Mihailetchi, and P. W. M. Blom, Appl. Phys. Lett. 88, 052104 (2006).

${ }^{34}$ M. M. Mandoc, L. J. A. Koster, and P. W. M. Blom, Appl. Phys. Lett. 90, 133504 (2007)

${ }^{35}$ For the case of classical $p n$ junctions, see J. Mattheis, J. H. Werner, and U. Rau, Phys. Rev. B 77, 085203 (2008).

${ }^{36}$ X. Yang, J. K. van Duren, R. A. J. Janssen, M. A. J. Michels, and J. Loos, Macromolecules 37, 2151 (2004).

${ }^{37}$ X. Yang, J. Loos, S. C. Veenstra, W. J. H. Verhees, M. M. Wienk, J. M. Kroon, M. A. J. Michels, and R. A. J. Janssen, Nano Lett. 5, 579 (2005).

${ }^{38}$ P. Peumans, A. Yakimov, and S. R. Forrest, J. Appl. Phys. 93, 3693 (2003).

${ }^{39}$ D. J. D. Moet, L. H. Slooff, J. M. Kroon, S. S. Chevtchenko, J. Loos, M. M. Koetse, J. Sweelssen, and S. C. Veenstra, MRS Symposia Proceedings No. 974 (Materials Research Society, Pittsburgh, 2007), Paper No. CC0309 .

${ }^{40}$ J. W. Eaton, GNU Octave Manual (Network Theory, Bristol, 2007). 